XL. (1983)

\title{
Global inverse problem of the Sturm-Liouville type for a linear elliptic partial differential equation of second order
}

\author{
by JAN BOCHENEK (Kraków)
}

\begin{abstract}
The purpose of the present paper is to generalize the known results on the so-called inverse problem of the Sturm-Liouville type (see [4], [5], [12], [13]). In particular, we deal in this paper with a certain theorem of Ambarzumian [1] and its generalizations (see [4], [5], [12]). The main purpose of this paper is to transfer the above Ambarzumian's theorem known for the Neumann boundary condition to other homogeneous boundary conditions. The method of this paper is based on the results of papers [4], [5], [12].
\end{abstract}

Introduction. Let $D$ be a bounded domain in the space $E^{m}$ with a sufficiently regular boundary $\partial D$. We assume that the boundary $\partial D$ of $D$ is so regular that there exists a Green function and sequences of eigenvalues and eigenfunctions for the value problem which is treated below. In the sequel we denote by $X=\left(x_{1}, \ldots, x_{m}\right)$ and $Y=\left(y_{1}, \ldots, y_{m}\right)$ the points of $E^{m}$.

We shall consider the problem of eigenvalues and eigenfunctions for the differential equation of the form

$$
L(u)+(\lambda-q) u=0
$$

with the boundary condition

$$
\frac{d u}{d v}-h u=0 \quad \text { on } D-\Gamma, \quad u=0 \quad \text { on } \Gamma,
$$

where

$$
\begin{gathered}
L(u)=\sum_{i, j=1}^{m} a_{i j} \frac{\partial^{2} u}{\partial x_{i} \partial x_{j}}, \\
\frac{d u}{d \nu}=\sum_{i, j=1}^{m} a_{i j} \frac{\partial u}{i x_{j}} \cos \left(n, x_{i}\right),
\end{gathered}
$$

$n$ being the interior normal to $\partial D$, and $\Gamma$ denotes an $(m-1)$-dimensional part of $\partial D$ ( $\Gamma$ being connected or not). We assume that $a_{i j}=a_{j i}(i, j=1, \ldots, m)$ are 
constant, the quadratic form $\sum_{i, j=1}^{m} a_{i j} \xi_{i} \xi_{j}$ is positive definite, $q$ is a continuous function and bounded in $D$ and $h \geqslant 0$ is a continuous function on $\partial D$. The boundary condition (2) may be taken in the sense of generalization (cf. [3]).

1. The Green function and eigenvalues and eigenfunctions of problem (1) (2). Let us denote by $G(X, Y, \lambda)$ the Green function and by $\left\{\lambda_{n}\right\},\left\{\varphi_{n}\right\}$ the sequences of eigenvalues and eigenfunctions of problem (1) (2), respectively. We assume that $\lambda \neq \lambda_{n}(n=1,2,3, \ldots)$ and that $\left\{\varphi_{n}\right\}$-form an orthonormal set of functions in $D$. We denote by $G^{(n)}(X, Y, \lambda)$ the iterated Green functions. For a definition and fundamental properties of the functions $G^{(n)}(X, Y, \lambda)$ see [14] or [4]. In the sequel by $G_{0}(X, Y, \lambda)$ we shall mean the Green function of the whole $E^{m}$ with respect to equation (1) when $q(X)=0$ for $X \in D$.

Let us assume that the parameter $\lambda$ in equation (1) is a real negative number and write $\varrho=-\lambda$, where $\varrho>0$. It is known (see [2] and [14]) that

$$
G_{0}(X, Y,-\varrho)=\sqrt{a}(2 \pi)^{-m / 2} r^{-(m / 2-1)} \varrho^{m / 4-1 / 2} K_{m / 2-1}(r \sqrt{\varrho}),
$$

where

$$
r^{2}=\sum_{i, j=1}^{m} A_{i j}\left(x_{i}-y_{i}\right)\left(x_{j}-y_{j}\right), \quad a=\operatorname{det}\left\|a_{i j}\right\|
$$

$A_{i j}$ are the elements of the inverse matrix to the matrix $\left\|a_{i j}\right\|$ and $K_{m / 2-1}$ is the modified Bessel function of the second kind of $\left(\frac{1}{2} m-1\right)$-th order.

Using the properties of the Green functions $G(X, Y, \lambda)$ and $G_{0}(X, Y, \lambda)$ and the form of $G_{0}(X, Y, \lambda)$ and its iterated Green functions and using also the asymptotic distribution of eigenvalues of problem (1)(2), we can prove the following formula:

$$
\sum_{n=1}^{\infty} \frac{\psi_{n}^{2}(X)}{\left(\mu_{n}+\varrho\right)^{k+1}}=\frac{1}{k !}\left\{\sqrt{a}(2 \pi)^{-m} \Gamma(k+1-m / 2) \varrho^{m / 2-k-1}-\Phi_{k}(X, \varrho)\right\} .
$$

The proof of formula (5) is quite similar to the proof of an analogous formula in [4]. In formula (5) $\left\{\mu_{n}\right\}$ and $\left\{\psi_{n}\right\}$ denote the sequences of eigenvalues and eigenfunctions of problem (1) (2), respectively, in the case $q=0$ in $D$ and $\Phi_{k}$ is a continuous function in $D$ for each $\varrho>0$ satisfying the condition

$$
\int_{L}\left|\Phi_{k}(X, \varrho)\right| d X=o\left(\varrho^{m / 2-k-1}\right) \quad \text { for } \varrho \rightarrow+\infty
$$

where $k$ in (5) and (6) is a positive integer such that $k \geqslant m / 2$.

It follows from (5) by Dini's well-known theorem that the series on the left-hand of (5) is uniformly convergent in the domain $D$. 
Therefore integrating over the domain $D$, we have

(7)

$$
\begin{aligned}
& \sum_{n=1}^{\infty} \frac{1}{\left(\mu_{n}+\varrho\right)^{k+1}} \\
& \quad=\frac{|D|}{k !} \sqrt{a}(2 \pi)^{-m} \Gamma(k+1-m / 2) \varrho^{m / 2-k-1}-\frac{1}{k !} \int_{\nu} \Phi_{k}(X, \varrho) d X,
\end{aligned}
$$

where $|D|$ is a measure of $D$.

2. Some properties of the trace of a linear operator. In this section we shall give a definition of the trace of a linear operator and we shall cite some lemmas and theorems, which will be used in the sequel.

The trace of a linear operator $A$ on a Hilbert space is the series

$$
\sum_{n=1}^{\infty}\left(A x_{n}, x_{n}\right)
$$

where $\left\{x_{n}\right\}$ denotes any complete orthonormal system of vectors in $H$ (cf. [8], p. 125).

If series (8) is convergent for any $\left\{x_{n}\right\}$, we say that $A$ has a finite trace. In the sequel the trace of the operator $A$ will be denoted by $S(A)$. If the operator $A$ has a finite trace, then sum (8) is independent of $\left\{x_{n}\right\}$ and series (8), converges absolutely for each $\left\{x_{n}\right\}$.

If the operator $A$ is positive, then sum (8) has the same value (finite or not) for any $\left\{x_{n}\right\}$ (cf. [8], p. 126-127).

Let $B$ be a completely continuous operator on $H$. Let us write $C$ $=\left(B^{*} B\right)^{1 / 2}$, where $B^{*}$ is the adjoint operator to $B$. Evidently the operator $C$ is a completely continuous, self-adjoint and positive operator on $H$. We shall denote by $\left\{s_{n}(B)\right\}$ the decreasing sequence of all eigenvalues of $C$ with all multiplicities listed (cf. [8], p. 46). By $\sigma_{p}(p>0)$ we denote the class of completely continuous operators $B$ such that

$$
|B|_{p}=\left\{\sum_{n=1}^{\infty}\left[s_{n}(B)\right]^{p}\right\}^{1 / p}
$$

If $B \in \sigma_{p}$ and $K$ is a bounded operator on $H$, then $B K$ and $K B$ belong to $\sigma_{p}$, and $|B K|_{p} \leqslant\|K\||B|_{p}$ and $|K B|_{p} \leqslant\|K\||B|_{p}$.

In the sequel we shall make use the following lemmas (see [8], Ch. III).

LemMA 1. If the operators $B_{j} \in \sigma_{p_{j}}(j=1, \ldots, s)$ and if $\sum_{j=1}^{s} p_{j}^{-1}=p^{-1} \leqslant 1$, then operator $B=B_{1} B_{2} \ldots B_{s}$ belong to $\sigma_{p}$ and $|B|_{p} \leqslant\left|B_{1}\right|_{p_{1}} \ldots\left|B_{s}\right|_{p_{s}}$.

LEMMA 2. The necessary and sufficient condition for the existence of a finite trace of $B$ is that $B \in \sigma_{1}$ and then

$$
|S(B)| \leqslant|B|_{1}=\sum_{n=1}^{\infty} s_{n}(B)
$$


LEMMA 3. If $A$ and $B$ are the linear and bounded operators on $H$, such that $A B \in \sigma_{1}$ and $B A \in \sigma_{1}$, then

$$
S(A B)=S(B A) .
$$

Let us denote by $T$ a self-adjoint and positive operator and by $V$ a self-adjoint and bounded operator on $H$. By $T_{Q}$ we denote the operator $T+\varrho I$, where $I$ is the identity operator on $H$, and $\varrho$ is a real positive number such that the operators $T_{e}$ and $T_{e}+V$ are the positively defined operators on $H$. We assume that the operators $T_{e}^{-1}$ and $\left(T_{Q}+V\right)^{-1}$ are completely continuous operators. We shall denote by $\left\{\mu_{n}\right\}$ and $\left\{\lambda_{n}\right\}$ the increasing sequences of all eigenvalues of $T$ and $T+V$, respectively, and by $\left\{x_{n}\right\}$ and $\left\{y_{n}\right\}$ the corresponding orthonormal sequences of eigenvectors. It follows from these assumptions that $\lim \mu_{n}=\lim \lambda_{n}=+\infty$, and $\left\{x_{n}\right\}$ and $\left\{y_{n}\right\}$ form the complete orthonormal systems in $H$ (cf. [13], p. 579).

LеммA 4. If the series $\sum_{n=1}^{\infty} \frac{1}{\left(\mu_{n}+\varrho\right)^{k+1}}$ is convergent and $C_{1}, \ldots, C_{k}$ are bounded operators on $H$, then the operator $A=T_{g}^{-1} C_{1} T_{g}^{-1} \ldots T_{g}^{-1} C_{k} T_{\underline{g}}^{-1}$ has a finite trace and

$$
|S(A)| \leqslant\left\|C_{1}\right\| \ldots\left\|C_{k}\right\| \sum_{n=1}^{\infty} \frac{1}{\left(\mu_{n}+\varrho\right)^{k+1}} .
$$

LEMMA 5. If the series $\sum_{n=1}^{\infty} \frac{1}{\left(\mu_{n}+\varrho\right)^{k+1}}$ is convergent for sufficiently large $\varrho$, then the operator $T_{Q}^{-k}-\left(T_{q}+V\right)^{-k}$ has a finite trace and

$$
S\left[T_{\ell}^{-k}-\left(T_{Q}+V\right)^{-k}\right]=\sum_{n=1}^{\infty}\left\{\frac{1}{\left(\mu_{n}+\varrho\right)^{k}}-\frac{1}{\left(\lambda_{n}+\varrho\right)^{k}}\right\} .
$$

The proofs of Lemmas 4 and 5 are given in paper [5].

We shall need the following assumption

Hypothesis $Z$. The sequence of eigenvalues $\left\{\mu_{n}\right\}$ of the operator $T$ satisfies the following condition

$$
\mu_{n}=C n^{x}+o\left(n^{x}\right) \quad \text { when } n \rightarrow+\infty,
$$

where $C$ and $\alpha$ are the positive constants independent of $n$.

We shall prove the following

THEOREM 1. If $1^{\circ}$ the series $\sum_{n=1}^{\infty} \frac{\left|\lambda_{n}-\mu_{n}\right|}{n}$ is convergent, $2^{\circ} k$ denotes the natural number such that $k \geqslant 1 / \alpha$, then

$$
\lim _{e \rightarrow \infty} \varrho^{k+1-1 / x} S\left[T_{e}^{-k}-\left(T_{e}+V\right)^{-k}\right]=0
$$


Proof. From the assumption $k \geqslant 1 / \alpha$ follows that the assumptions of Lemma 5 are fulfilled. Using (11) we need to prove that

$$
\lim _{e^{\rightarrow+\infty}} \varrho^{k+1-1 / \alpha} \sum_{n=1}^{\infty}\left\{\frac{1}{\left(\mu_{n}+\varrho\right)^{k}}-\frac{1}{\left(\lambda_{n}+\varrho\right)^{k}}\right\}=0 .
$$

Let us observe that

$$
\begin{aligned}
\varrho^{k+1-1 / \alpha} \sum_{n=1}^{\infty}\{ & \left.\frac{1}{\left(\mu_{n}+\varrho\right)^{k}}-\frac{1}{\left(\lambda_{n}+\varrho\right)^{k}}\right\} \\
= & \sum_{n=1}^{\infty} \frac{\lambda_{n}-\mu_{n}}{n} \frac{n}{\left(\mu_{n}+\varrho\right)^{1 / \alpha}}\left(\frac{\varrho}{\mu_{n}+\varrho}\right)^{k-1 / \alpha} \times \\
& \times \frac{\left[\left(\lambda_{n}+\varrho\right)^{k-1}+\left(\lambda_{n}+\varrho\right)^{k-2}\left(\mu_{n}+\varrho\right)+\ldots+\left(\mu_{n}+\varrho\right)^{k-1}\right] \varrho}{\left(\lambda_{n}+\varrho\right)^{k}} .
\end{aligned}
$$

Because the sequences

$$
\begin{aligned}
\left\{\frac{n}{\left(\mu_{n}+\varrho\right)^{1 / \alpha}}\right\}, & \left\{\left(\frac{\varrho}{\mu_{n}+\varrho}\right)^{k-1 / x}\right\} \\
\text { and } \quad & \left\{\frac{\left[\left(\lambda_{n}+\varrho\right)^{k-1}+\left(\lambda_{n}+\varrho\right)^{k-2}\left(\mu_{n}+\varrho\right)+\ldots+\left(\mu_{n}+\varrho\right)^{k-1}\right] \varrho}{\left(\lambda_{n}+\varrho\right)^{k}}\right\}
\end{aligned}
$$

are uniformly bounded in $\varrho$ for $\varrho>0$ and $n \in N$, from the least equality follows that the series

$$
M \sum_{n=1}^{\infty} \frac{\left|\lambda_{n}-\mu_{n}\right|}{n}
$$

is an absolute majorant of the series

$$
\sum_{n=1}^{\infty} \varrho^{k+1-1 / \alpha}\left\{\frac{1}{\left(\mu_{n}+\varrho\right)^{k}}-\frac{1}{\left(\lambda_{n}+\varrho\right)^{k}}\right\}
$$

where $M$ is a positive constant independent of $\varrho>0$ and $n \in N$.

Consequently this series is absolutely and uniformly convergent in $\varrho$ for $\varrho>0$. Therefore from this we get

$$
\lim _{\varrho \rightarrow \infty} \varrho^{k+1-1 / \alpha} \sum_{n=1}^{\infty}\left\{\frac{1}{\left(\mu_{n}+\varrho\right)^{k}}-\frac{1}{\left(\lambda_{n}+\varrho\right)^{k}}\right\}=0 .
$$

From (15) by (11) follows (13).

As we proved in paper [5], under assumption $\mu_{1}+\varrho>\|V\|$, the following equality holds

$$
T_{Q}^{-k}-\left(T_{Q}+V\right)^{-k}=B_{Q}+F_{Q},
$$


where

$$
B_{Q}=T_{\dot{e}}^{-k} V T_{e}^{-1}+\ldots+T_{Q}^{-1} V T_{e}^{-k}
$$

and

$$
\left|S\left(F_{e}\right)\right| \leqslant \frac{K}{\mu_{1}+\varrho} \sum_{n=1}^{\infty} \frac{1}{\left(\mu_{n}+\varrho\right)^{k+1}},
$$

where $K$ is a positive constant independent of $\varrho$.

THEOREM 2. Under the assumptions of Theorem 1 we have the equality

$$
\lim _{\ell \rightarrow \infty} \varrho^{k+1-1 / x} S\left(V T_{Q}^{-k-1}\right)=0 .
$$

Proof. At first let us observe that from assumption $k \geqslant 1 / \alpha$, and because $V$ is a bounded operator, we have $V T_{e}^{-k-1} \in \sigma_{1}$ for $\varrho>0$. From this by Lemma 2 we get that the operator $V T_{e}^{-k-1}$ has the finite trace. Analogously, we prove that also the operator $B_{\ell}$ for $\varrho>0$ has a finite trace. Therefore

and

$$
S\left(V T_{e}^{-k-1}\right)=\sum_{n=1}^{\infty}\left(V T_{Q}^{-k-1} x_{n}, x_{n}\right)=\sum_{n=1}^{\infty} \frac{\left(V x_{n}, x_{n}\right)}{\left(\mu_{n}+Q\right)^{k+1}}
$$

$$
\begin{aligned}
S\left(B_{g}\right) & =\sum_{n=1}^{\infty}\left(B_{\underline{q}} x_{n}, x_{n}\right)=\sum_{n=1}^{\infty}\left(T_{\underline{q}}^{-k} V T_{\underline{u}}^{-1} x_{n}, x_{n}\right)+\ldots+\sum_{n=1}^{\infty}\left(T_{\underline{q}}^{-1} V T_{\underline{q}}^{-k} y_{n}, x_{n}\right) \\
& =k \sum_{n=1}^{\infty} \frac{\left(V x_{n}, x_{n}\right)}{\left(\mu_{n}+Q\right)^{k+1}} .
\end{aligned}
$$

Hence

$$
S\left(V T_{e}^{-k-1}\right)=\frac{1}{k} S\left(B_{e}\right)
$$

On the other hand from inequality (18) and from this that the series

$$
\sum_{n=1}^{\infty} \varrho^{k-1 / x} \frac{1}{\left(\mu_{n}+\varrho\right)^{k+1}}
$$

is uniformly convergent in $\varrho$ for $\varrho>0$, follows the equality

$$
\lim _{\varrho \rightarrow \infty} \varrho^{k+1-1 / x} S\left(F_{\varrho}\right)=0 \text {. }
$$

Because by (16) we have

$$
S\left(B_{Q}\right)=S\left[T_{Q}^{-k}-\left(T_{Q}+V\right)^{-k}\right]-S\left(F_{Q}\right),
$$

then from this and from equalities (13), (20) and (21) follows (19). This yields Theorem 2 . 
3. Dependence of the function $q$ on the eigenvalues of problem (1) (2). In the domain $D$ defined in the introduction of this paper we shall consider the problem of the eigenvalues and eigenfunctions for equation (1) with boundary condition (2).

Let $\left\{\lambda_{n}\right\}$ denote the increasing sequence of eigenvalues of problem (1) (2) and let $\left\{\varphi_{n}\right\}$ denote the corresponding orthonormal sequence of eigenfunctions of this problem. Further, by $\left\{\mu_{n}\right\}$ and $\left\{\psi_{n}\right\}$ we denote the sequences of eigenvalues and eigenfunctions of problem (1) (2) in the case $q=0$ in $D$.

We shall prove the following

THEOREM 3. If $q$ is a continuous and bounded function in $D$ the series $\sum_{n=1}^{\infty} \frac{\left|\lambda_{n}-\mu_{n}\right|}{n}$ is convergent, then

$$
\int_{D} q(X) d X=0
$$

Proof. Let $H=L_{2}(D)$ be a Hilbert space of functions square-sumable on $D$ and let $T$ be a self-adjoint operator on $H$, which is a Fridrichs expansion of the operator $-L$ defined by (3), while $V$ is multipler operator $q$. From equality (7) follows that

$$
\mu_{n}=C n^{2 / m}+o\left(n^{2 / m}\right), \quad \text { where } n \rightarrow+\infty,
$$

where $C$ is a positive constant independent on $n$, but $m$ is the dimmensional of the considered space $E^{m}$. From this follows that the assumptions of Theorem 2 are fulfilled, where $\alpha=2 / m, k \geqslant m / 2$. Therefore

$$
\lim _{\ell \rightarrow \infty} \varrho^{k+1-m / 2} S\left(V T_{e}^{-k-1}\right)=0 .
$$

We express the trace of the operator $V T_{Q}^{-k-1}$ by the sequence $\left\{\psi_{n}\right\}$. We obtain

$$
S\left(V T_{Q}^{-k-1}\right)=\sum_{n=1}^{\infty} \frac{\left(V \psi_{n}, \psi_{n}\right)}{\left(\mu_{n}+\varrho\right)^{k+1}}
$$

By the definition of the operator $V$ and by the uniform convergence of the series in formula (5) with respect to $X \in D$, equality (25) may be written in the form

$$
S\left(V T_{e}^{-k-1}\right)=\left(q, \sum_{n=1}^{\infty} \frac{\psi_{n}^{2}}{\left(\mu_{n}+\varrho\right)^{k+1}}\right)
$$

By (5) equality (26) take the form

$$
S\left(V T_{\varrho}^{-k-1}\right)=\frac{1}{k !}\left\{\sqrt{a}(2 \pi)^{-m} \Gamma(k+1-m / 2) \varrho^{m / 2-k-1}(q, 1)-\left(q, \Phi_{k}\right)\right\} .
$$


Because

$$
\left|\left(q, \Phi_{k}\right)\right|=\left|\int_{D} q(X) \Phi_{k}(X, \varrho) d X\right| \leqslant \max _{x \in D}|q(X)| \int_{\nu}\left|\Phi_{k}(X, \varrho)\right| d X
$$

by (6) we get

$$
\left(q, \Phi_{k}\right)=o\left(\varrho^{m / 2-k-1}\right), \quad \text { where } \varrho \rightarrow \infty .
$$

By (24), (27) and (28) we obtain

$$
\lim \frac{1}{k !}\left\{\sqrt{a}(2 \pi)^{-m} \Gamma(k+1-m / 2)(q, 1)+o(1)\right\}=0 .
$$

Equality (29) is posible only when

$$
(q, 1)=0 \text {. }
$$

It is obvious that (30) is equivalent to equality (22). The proof of Theorem 3 is completed.

Remark 1. From Theorem 3 under the assumption

$$
\forall X \in D q(X) \geqslant 0 \text {, }
$$

follows the equality

$$
\forall X \in D q(X)=0 .
$$

As we prove in paper [5], if we consider equation (1) with the boundary condition of Neumann type, i.e.,

$$
\frac{d u}{d v}=0 \quad \text { on } \partial D
$$

and if the function $q$ satisfies equality (22), then the sufficient condition for (32) is $\lambda_{1} \geqslant 0$, where $\lambda_{1}$ is the first eigenvalue of problem (1) (33).

Remark 2. The assumption $\lambda_{1} \geqslant 0$, where $\lambda_{1}$ is the first eigenvalue of problem (1) (33), is equivalent to the assumption $\bar{\lambda}_{1} \geqslant 0$, where $\bar{\lambda}_{1}$ is the first eigenvalue for the equation

$$
\Delta u+(\lambda-q) u=0 \quad \text { in } D
$$

with boundary condition (33).

From the variational definition of the eigenvalues and eigenfuntions of problem (34) (33), follows that the first eigenvalue $\bar{\lambda}_{1} \geqslant 0$ if and only if

$$
\forall \varphi \in C_{\sigma}^{1}(D) \int_{D}\left[\operatorname{grad}^{2} \varphi(X)+q(X) \varphi^{2}(X)\right] d X \geqslant 0 .
$$

Let us denote by $Q^{+}(D)$ the set of all functions $q$ which are continuous and bounded in $D$ and satisfy condition (35). 
Remark 3. It is obvious that $Q^{+}(D)$ contains the all non-negative continuous and bounded functions in domain $D$. In section 4 we shall prove that set $Q^{+}(D)$ contains also some functions which change their sing in $D$. THEOREM 4. If the function $q \in Q^{+}(D)$ and the series $\sum_{n=1}^{\infty} \frac{\left|\lambda_{n}-\mu_{n}\right|}{n}$ is
convergent, then $q(X)=0$ for each $X \in D$.

Proof. From the fact that the assumptions of this theorem imply the assumptions of Theorem 3, we obtain equality (22). On the other hand because $q \in Q^{+}(D)$ by $(35)$ and variational definition of eigenvalues and eigenfunctions of problem (34) (33), we get

$$
\bar{\lambda}_{1}=\min _{\varphi \in C_{\sigma}^{1}(D)} \int_{D}\left[\operatorname{grad}^{2} \varphi(X)+q(X) \varphi^{2}(X)\right] d X \geqslant 0,
$$

where

$$
\int_{D} \varphi^{2}(X) d X=1
$$

Since $q$ satisfies (22), then function $\varphi_{1}=|D|^{-1 / 2}$ realizes the minimum (36), and $\bar{\lambda}_{1}=0$. From this follows that for problem (34) (33) the first eigenvalue $\bar{\lambda}_{1}=0$ and its corresponding first eigenfunction is $\varphi_{1}=|D|^{-1 / 2}=$ const. Therefore the function $\varphi_{1}=|D|^{-1 / 2}$ satisfies equation (34) with $\lambda=0$ and the boundary condition (33) (cf. [6]). From this we get

$$
\forall X \in D q(X)=0 \text {. }
$$

COROLlaRY 1. From Theorem 4 follows that if the function $q$ in equation (1) belongs to $Q^{+}(D)$ and if the sequence $\left\{\lambda_{n}\right\}$ of eigenvalues of problem (1) (2) is near to the sequence $\left\{\mu_{n}\right\}$ of eigenvalues of equation

$$
L(u)+\lambda u=0
$$
with the boundary condition (2), in this sense that the series $\sum_{n=1}^{\infty} \frac{\left|\lambda_{n}-\mu_{n}\right|}{n}$ is
convergent, then problems (1) (2) and (38) (2) are identical.

4. Positiveness of the operator $T+V$. From the assumption $h(X) \geqslant 0$ for $X \in \partial D$ and from the dependence of the eigenvalues on the boundary conditions follows that if $q \in Q^{+}(D)$, then the sequence of eigenvalues of problem (1) (2) is an increasing sequence of positive numbers. This means that the operator $T+V$, defined in section 3 , is positive operator. On the other hand the operator $T$ is also positive operator. The purpose of this section is to prove that from the fact that $T$ and $T+V$ are positive operators does not follow that $V$ is a positive operator, where $T$ and $V$ are the operators defined in section 3. In other words we shall prove that from the fact that $q \in Q^{+}(D)$ does not follow that $q(X) \geqslant 0$ for all $X \in D$. To simply the consideration we assume that $m=1, D=(0, \pi)$ and

$$
L=d^{2} / d x^{2},
$$


then condition (35) takes the form

$$
\forall \varphi \in C_{\sigma}^{1}(0, \pi) \int_{0}^{\pi}\left\{\left[\varphi^{\prime}(x)\right]^{2}+q(x) \varphi^{2}(x)\right\} d x \geqslant 0
$$

Let us put $q_{\varepsilon}(x):=x-\varepsilon$, where $\varepsilon$ is a positive constant such that $0<\varepsilon<\pi / 4$. Let us denote

$$
\lambda_{1}(\varepsilon)=\min _{\varphi \in C_{\sigma}^{1}(0, \pi)}\left\{\int_{0}^{\pi}\left\{\left[\varphi^{\prime}(x)\right]^{2}+q_{\varepsilon}(x) \varphi^{2}(x)\right\} d x: \int_{0}^{\pi} \varphi^{2}(x) d x=1\right\}
$$

and by $\varphi_{\varepsilon}$ the function which realizes the minimum (39). As we know $\varphi_{\varepsilon}(x) \neq 0$ for all $x \in(0, \pi)$, and by (39) $\int_{0}^{\pi} \varphi_{\varepsilon}^{2}(x) d x=1$ for every $\varepsilon>0$.
Therefore

$$
\begin{aligned}
\lambda_{1}(\varepsilon) & =\int_{0}^{\pi}\left\{\left[\varphi_{\varepsilon}^{\prime}(x)\right]^{2}+q_{\varepsilon}(x) \varphi_{\varepsilon}^{2}(x)\right\} d x \\
& \geqslant \int_{0}^{\varepsilon}\left\{\left[\varphi_{\varepsilon}^{\prime}(x)\right]^{2}-\varepsilon \varphi_{\varepsilon}^{2}(x)\right\} d x+\int_{\varepsilon}^{3 \varepsilon}\left[\varphi_{\varepsilon}^{\prime}(x)\right]^{2} d x+\int_{3 \varepsilon}^{\pi}\left\{\left[\varphi_{\varepsilon}^{\prime}(x)\right]^{2}+2 \varepsilon \varphi_{\varepsilon}^{2}(x)\right\} d x \\
& \geqslant-\varepsilon \int_{0}^{\varepsilon} \varphi_{\varepsilon}^{2}(x) d x+2 \varepsilon \int_{3 \varepsilon}^{\pi} \varphi_{\varepsilon}^{2}(x) d x \\
& =\varepsilon\left[2 \int_{3 \varepsilon}^{\pi} \varphi_{\varepsilon}^{2}(x) d x-\int_{0}^{\varepsilon} \varphi_{\varepsilon}^{2}(x) d x\right] .
\end{aligned}
$$

Because

$$
\lim _{\varepsilon \rightarrow 0} \int_{3 \varepsilon}^{\pi} \varphi_{\varepsilon}^{2}(x) d x=1
$$

there exists $\varepsilon_{0}>0$ such that for $0<\varepsilon \leqslant \varepsilon_{0}$ we have

$$
\int_{3 \varepsilon}^{\pi} \varphi_{\varepsilon}^{2}(x) d x \geqslant \frac{2}{3}
$$

From this follows that

$$
\int_{0}^{\varepsilon} \varphi_{\varepsilon}^{2}(x) d x \leqslant \frac{1}{3}
$$

By (40) and (41) we get

$$
\lambda_{1}(\varepsilon) \geqslant \varepsilon \quad \text { for } 0<\varepsilon \leqslant \varepsilon_{0} .
$$

This means that the function $q_{\varepsilon}$ such that $q_{x}(x)=-x-\varepsilon$ for $0<\varepsilon \leqslant \varepsilon_{0}$ belongs to $Q^{+}(0, \pi)$ and $q_{\varepsilon}$ changes its sign in the interval $(0, \pi)$. 


\section{References}

[1] W. A. Ambarzumian, Über eine Frage der Eigenwertteorie, Zeits, für Physik 53 (1929), p. 690-695.

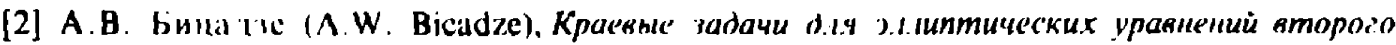
nop:t)кa. (Boundary raluc problems for elliptic ('quations of second order), Moscow 1966.

[3] J. Bochenek, On some problems in the theory of eigenvalues and eigenfunctions associated with linear elliptic partial differential equations of the second order, Ann. Polon. Math. 16 (1965), p. 153-167.

[4] - Zależność równania różniczkowego czqstkowego od wartości wlasnych odpowiedniego zagadnienia, Zeszyty Naukowe Politechniki Krakowskiej z. 15 (1968), p. 3-54.

[5] - On the inverse problem of the Sturm-Liouville type for a linear elliptic partial differential equation with constant coefficients of the second order, Ann. Polon. Math. 24 (1971), p. 331-341.

[6] R. Courant and D. Hilbert, Methods of mathematical physics I, New York 1953.

[7] K. Fan, On a theorem of Veyl concerning eigenvalues of linear transformations I, Proc. Acad. Nat. Sci. 35 (1945), p. 652-655.

[8] И.Ц. Гохберг и М.Г. Крейн (I. С. Gohberg and M. G. Krein), Введение в теорию линейных несамосопряженных операторов в гилбертовом пространстве. (Introduction to the theory of non-self-adjoint operators in Hilbert space), Moscow 1965.

[9] K. Knopp, Szeregi nieskończone, Warszawa 1956.

[10] M. Krzyżański, Partial differential equations of second order $I, I I$, Warszawa 1971.

[11] Н.Н. Кузисцов (N. W. Kuznecov), Обоб́щепие одной теоремы Либариумяна, (A generalization of a theorem of Libarcumian), Dokl. Akad. Nauk SSSR 146, 6 (1962), p. $1259-1262$.

[12] Б.М. Левитан и М.Г. Гасымов (В. М. Levitan and M. G. Gasymov), Onpedenenue дифференциального уравнения по двум спектрам (Defining a differential equation by two spectra), Uspehi Mat. Nauk 19.2 (1964), p. 3-63.

[13] В.И. Смирнов (W. I. Smirnov), Курс высшей математики V, (A course of higher mathematics), vol. V, Moscow 1959.

[14] E. C. Titchmarsh, Eigenfunctions expansions associated with second order differential equations II, Oxford 1958.

Reçu par la Rédaction le 20. 5. 1978 